\title{
Regulator of G protein-signaling proteins and addictive drugs
}

\author{
John Traynor \\ Department of Pharmacology and Substance Abuse Research Center, University of Michigan, Ann Arbor, Michigan, USA \\ Address for correspondence: J. R. Traynor, Department of Pharmacology, University of Michigan, Ann Arbor, MI 48109-5632. \\ Voice: 734-7647-7479; fax: 734-763-4450. jtraynor@umich.edu
}

\begin{abstract}
Regulator of G protein-signaling (RGS) proteins are a family of more than 30 intracellular proteins that negatively modulate intracellular signaling of receptors in the $G$ protein-coupled receptor family. This family includes receptors for opioids, cannabinoids, and dopamine that mediate the acute effects of addictive drugs or behaviors and chronic effects leading to the development of addictive disease. Members of the RGS protein family, by negatively modulating receptor signaling, influence the intracellular processes that lead to addiction. In turn, addictive drugs control the expression levels of several RGS proteins. This review will consider the distribution and mechanisms of action of RGS proteins, particularly the R4 and R7 families that have been implicated in the actions of addictive drugs, how knowledge of these proteins is contributing to an understanding of addictive processes, and whether specific RGS proteins could provide targets for the development of medications to manage and/or treat addiction.
\end{abstract}

Keywords: drugs of abuse; opioids; stimulants; opioid receptors; dopamine receptors; RGS proteins; G proteins; cell signaling

\section{Introduction}

Regulator of $\mathrm{G}$ protein-signaling (RGS) proteins are a family of more than 30 intracellular proteins that negatively modulate signaling pathways of $G$ protein-coupled receptors (GPCRs). ${ }^{1-4}$ GPCRs are a large family of plasma membrane receptors for endogenous neurotransmitters that couple to intracellular heterotrimeric GTP binding proteins (G proteins) comprising an $\alpha$ subunit and a heterodimer of $\beta \gamma$ subunits, which activate signaling cascades within the cell. GPCRs are responsible for the actions of many different addictive substances either as direct targets or as a secondary consequence of the action of the addictive agent. These receptors and their cognate signaling pathways not only mediate acute effects of addictive drugs/behaviors but are also vital to the development of the addictive disease whether this be addiction to legal (alcohol, nicotine) or illegal (amphetamine, cocaine, heroin, cannabis) drugs, substances (solvents), or behavioral addictions (gambling). Because RGS proteins modulate GPCR signaling, they are important components in the control of intracellular events that drive the addictive process. Indeed, the interaction between addictive drugs and RGS proteins appears to be interdependent because there is considerable evidence that RGS proteins are in turn manipulated by the addictive process. ${ }^{5}$ Although it can be hypothesized that RGS proteins play a role in all addictions, published literature to date has concentrated on the stimulants amphetamine and cocaine and the opioid morphine.

\section{RGS protein modulation of G protein signaling}

Members of the GPCR family of receptors include the opioid receptors, especially the $\mu$-opioid receptor as the primary target for heroin and prescription opioids, the cannabinoid CB1 receptors, the dopamine D1 and D2 families of receptors that are the mediators of reward or the anticipation of reward for many addictions ${ }^{6,7}$ as well as metabotropic glutamate receptors that may regulate many behavioral actions of addictive drugs. ${ }^{8}$

GPCRs show specificity for coupling to a particular type of $\mathrm{G} \alpha$ protein. $\mathrm{G} \alpha$ proteins are 


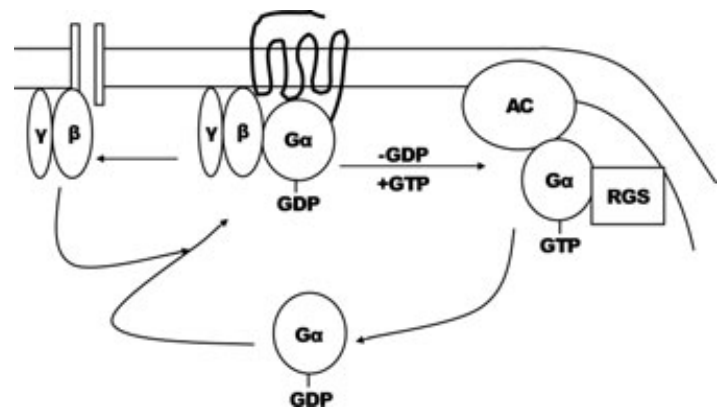

Figure 1. G protein cycle. In the resting state the seventransmembrane domain receptor is associated with the $\mathrm{G} \boldsymbol{\alpha} \boldsymbol{\beta} \boldsymbol{\gamma}$ heterotrimeric $\mathrm{G}$ protein with the $\mathrm{G} \boldsymbol{\alpha}$ subunit bound to GDP. Activation of the receptor promotes the exchange of GDP for GTP on the G $\alpha$ subunit. This leads to separation of the $G \boldsymbol{\alpha}$ and $G \boldsymbol{\beta} \gamma$ subunits and allows them to interact with downstream effectors, such as adenylyl cyclase (AC) and ion channels. The pool of active G $\boldsymbol{\alpha}-\mathrm{GTP}$ is slowly hydrolyzed by the GTPase activity of the $\mathrm{G} \boldsymbol{\alpha}$ subunit. This process is accelerated by the GAP activity of RGS proteins. The inactive G $\alpha-G D P$ has high affinity for the $G \boldsymbol{\beta} \boldsymbol{\gamma}$ subunits, the heterotrimer is reformed, and the cycle can start again. By reducing the lifetime of the active $\mathrm{G} \boldsymbol{\alpha}-\mathrm{GTP}$, RGS proteins also reduce the accumulation of the $G \boldsymbol{\beta} \boldsymbol{\gamma}$ subunits and so negatively modulate all branches of $G$ protein-mediated signaling.

divided into four broad families: Gos activates the enzyme adenylyl cyclase leading to an increase in cAMP in the cell, the Gai/o family inhibits adenylyl cyclase, the Goq family activates the enzyme phospholipase $\mathrm{C}$, leading to the release of calcium from intracellular stores, and $\mathrm{G} \alpha_{12 / 13}$ regulates cell growth and differentiation. The associated $\beta \gamma$ subunits also interact with a variety of intracellular effectors including inwardly rectifying potassium channels, calcium channels, phospholipase C, and the mitogen-activated protein kinase pathway. Dopamine D1 receptors primarily couple to Gos, the opioid, cannabinoid, dopamine D2 receptors, and metabotropic glutamate receptors 2 and 3 pre-

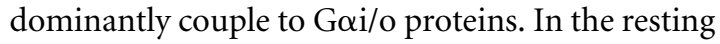
state, the $\mathrm{G} \alpha$ of the heterotrimer $\mathrm{G}$ protein binds the guanine nucleotide GDP. Occupation of a GPCR by endogenous neurotransmitters or exogenous agonists activates the receptor, triggering the exchange of GDP for GTP on the G $\alpha$ subunit and separation from the $\beta \gamma$ subunits. Both $\mathrm{G} \alpha-\mathrm{GTP}$ and $\beta \gamma$ activate downstream effectors. Hydrolysis of the bound
GTP by the GTPase activity of the $\alpha$ subunit provides $\mathrm{G} \alpha-\mathrm{GDP}$, which re-associates with the $\beta \gamma$ heterodimers thus terminating the signal. RGS proteins bind to the GTP-bound G $\alpha$ subunit and accelerate the rate of hydrolysis of GTP (Fig. 1).

RGS proteins are characterized by the presence of a consensus 125 amino-acid sequence, the so-called RGS box or RH homology domain (Fig. 2), which binds $\mathrm{G} \alpha-\mathrm{GTP}$ proteins ${ }^{1-4,9}$ and functions as a GAP

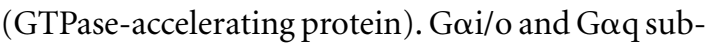
types of $\mathrm{G} \alpha$ proteins are sensitive to this GAP activity due to their relatively slow endogenous rate of GTP hydrolysis. In contrast, G $\alpha$ s, which already hydrolyses GTP rapidly, is insensitive to GAP activity. GAP

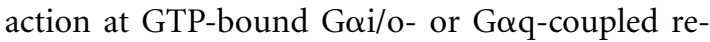
ceptors leads to a more rapid return to the inactive G $\alpha$-GDP state and a faster recombination of the $\mathrm{G} \alpha$ and $\beta \gamma$ signaling molecules. By reducing the lifetime of $G \alpha-G T P$ and $\beta \gamma$ molecules, RGS proteins limit $G$ protein signal strength at steady state and thus act as negative modulators of $G$ protein signaling. As a result, RGS proteins exhibit profound control over both the potency and efficacy of agonist action. For example, in an in vitro system completely lacking RGS activity at G $\alpha$ o, the efficacy of morphine is increased several-fold and the potency 40 -fold converting morphine from a partial agonist to a robust full agonist. ${ }^{10}$

\section{RGS protein families}

Many proteins with $\mathrm{RH}$ domains have been identified. These proteins have been divided into various families (Table 1) on the basis of similarity in amino acid composition. ${ }^{2,4}$ Members of the R4 and RZ families are smaller proteins consisting largely of

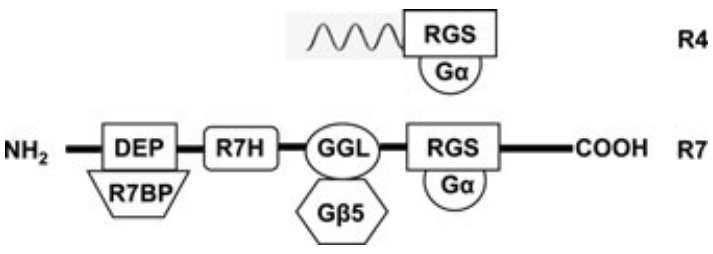

Figure 2. Cartoon of R4- and R7-type RGS proteins

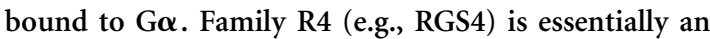
RGS domain plus an N-terminal helical domain. The more complex R7 family (e.g., RGS9-2) contains proteinprotein binding domains to allow association with other proteins, including the obligate partners R7 binding protein (R7BP) and G及5. For other abbreviations see Table 1. 
Table 1. Families of RGS proteins ${ }^{a}$

\begin{tabular}{lll}
\hline Family & \multicolumn{1}{c}{ Members } & Structure: RGS domain plus \\
\hline RZ & RGS17, $19,20^{b}$ & N-terminal cysteine string \\
R4 & RGS1, $2,3,{ }^{c} 4,5,8,13,16,18,21$ & N-terminal amphipathic helix \\
R7 & RGS6, $7,9,11$ & GGL + DEP + R7H domains \\
R12 & RGS10, 12, 14 & Variable-can contain PDZ \\
& & RBD, and GoLoco domains \\
RA & Axin, Conductin & GSK + CAT + PP2A + DIX \\
RL & P115RhoGEF & DH + PH \\
RL & GRK2 and 3 & Ser/Thr kinase + PH \\
\hline
\end{tabular}

${ }^{a} \mathrm{CAT}, \beta$-catenin binding site; DEP, disheveled, EGL-10, pleckstrin homology domain; DH, Dbl-homology (RhoGEF) domain; GEF, guanine-nucleotide exchange factor; GGL, G protein $\gamma$-like domain; GoLoco, G $\alpha_{\mathrm{i} / \mathrm{o}}-\mathrm{GDP}$ binding motif; GRK, G protein receptor kinase; GSK, glycogen synthase kinase $3 \beta$-binding domain; PDZ, PSD95/Dlg/Z0-1 domain; $\mathrm{PH}$, pleckstrin homology domain; R7H, R7 homology domain; PP2A, protein phosphatase 2A homology region; RBD, Rap1/2-binding domain; DIX, dimerization domain.

${ }^{b}$ RGS17 and 20 are also known as RGSZ2 and RGSZ1, respectively; RGS20 is also known as GAIP (G $\alpha$-interacting protein).

${ }^{c}$ RGS3 is much larger than other members of the R4 family and has multiple splice variants, some of which include a PDZ domain.

the RGS domain (Fig. 2). Other families have much more complex structures with domains that allow for protein-protein interactions. Particularly pertinent to addiction is RGS9-2 (Fig. 2), a member of the R7 family, which, in addition to the conserved RGS box, contains several other domains/motifs. R7 family members possess a DEP (disheveled, Egl10 , pleckstrin) domain that binds the adapter protein R7BP, ${ }^{11,12}$ which promotes membrane association $^{13,14}$ and stabilizes RGS9-2 against degradation. The R7 family members also have a GGL (G gamma like) domain that allows binding to the $G$ protein $\beta 5$ subunit (Gß5), ${ }^{15}$ which further promotes protein stability. ${ }^{16}$ RGS proteins may also be regulated by phosphorylation ${ }^{17,18}$ and lipid modification. ${ }^{19}$ Strongly acidic lipids, such as phosphatidyl inositol trisphosphate $\left(\mathrm{PIP}_{3}\right)$ and phosphatidic acid, transiently bind to and inhibit the GAP activity of R4 family members; an inhibition that can be reversed by calcium/calmodulin. ${ }^{20,21}$

In addition to RGS proteins that act as GAPs, there are other proteins with $\mathrm{RH}$ domains that bind to $\mathrm{G} \alpha$ but do not necessarily exhibit GAP activity. The RH domain of GPCR kinases (GRKs) that are recruited by $\beta \gamma$ subunits for receptor phosphorylation binds

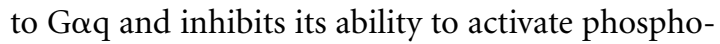
lipase C. ${ }^{22}$ The Rho guanine nucleotide exchange factors (RhoGEFs) bind to the $G$ proteins $G \alpha_{12}$ and
$\mathrm{G}_{13}{ }^{23}$ and also interact with G $\alpha \mathrm{q} .{ }^{24}$ The primary function of the RH domain in the RH-RhoGEF proteins is to link the GPCR to Rho-mediated signaling pathways that control many cell processes including the actin cytoskeleton, cell growth, proliferation, and differentiation. ${ }^{25}$ The proteins axin and conductin regulate the Wnt signaling pathway important for embryogenesis and cancer.

Although many RGS proteins are GAPs for all

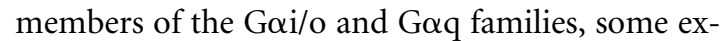
hibit selectivity. For example members of the R7 family, which includes RGS9-2, are selective for Goo, ${ }^{26,27}$ whereas RGS2 is considered to be more effective as a GAP at G $\alpha$ q. ${ }^{28}$ There is also increasing evidence that specificity for RGS proteins can be conferred by the GPCR. ${ }^{29-32}$ Several RGS proteins, including RGS2, 4, 8, and 9, have been shown to act as GAPs to modulate $\mu$-opioid receptor signaling in vitro, but there may be specificity under endogenous conditions. ${ }^{33}$ However, the distribution of RGS protein types relative to receptor and $\mathrm{G} \alpha$ subtype distribution may be the most important facet governing selectivity. ${ }^{5}$

\section{Distribution of RGS proteins}

mRNA for the various members of the RGS protein family is unevenly distributed across the central 
nervous system. In particular, there is specific localization of certain RGS proteins in regions implicated in the action of addictive drugs. RGS2 mRNA is found in the periform and neocortex, caudate putamen, amygdala, hippocampus, and locus coeruleus (LC). ${ }^{34,35}$ RGS4 mRNA is found in high levels in the thalamus, caudate putamen, and nucleus accumbens (NAcc), as well as the prefrontal cortex ${ }^{36,37}$ where incorrect dopamine and serotonin function may be responsible for some of the symptoms of schizophrenia. Indeed, RGS4 has been suggested as a susceptibility gene for schizophrenia ${ }^{38,39}$ and RGS4 protein is reduced in the prefrontal cortex of human schizophrenic post mortem brains ${ }^{40}$ and in a rat model of schizophrenia. ${ }^{41}$

RGS9 is found in two forms: a short form (RGS91) that is exclusive to the retina, where it acts as a GAP for the retinal G protein Gat, and a longer form (RGS9-2) that is largely confined to striatal regions in rodent and human brain, that is, the caudate putamen, NAcc, islands of Calleja, and olfactory tubercle. ${ }^{36,42-44}$ This distribution mimics that of striatal specific proteins, such as dopamine D1 receptors, adenylyl cyclase type $\mathrm{V}$, and the phosphoprotein DARPP-32 (dopamine and cyclic adenosine $3^{\prime}, 5^{\prime}$-monophosphate regulated phosphoprotein with molecular mass of $32 \mathrm{kDa}) .{ }^{45}$ In addition, RGS9-2 is expressed at lower levels in regions associated with the antinociceptive actions of morphine, namely the periaqueductal gray (PAG) and the dorsal horn of the spinal cord. ${ }^{46}$

\section{Interactions between RGS proteins and drugs of abuse}

The localization of RGS2 and 4 and in particular RGS9-2 led to speculation of a role in addictive processes. This idea is strengthened by several reports showing that addictive substances cause changes in the levels of RGS proteins or RGS mRNA, suggesting a role for these drug-induced modifications in the addictive process. Several studies have addressed changes in RGS after drug exposure but there have been fewer studies on the roles of RGS proteins in modulating responses to addictive drugs.

\section{RGS2}

The mRNA for RGS2 has a short half-life, ${ }^{47}$ and the RGS2 protein itself is targeted for proteasomal degradation, ${ }^{48}$ suggesting that RGS2 protein levels are tightly regulated. Although no studies to date have demonstrated changes in RGS2 protein in response to addictive drugs, there is evidence for alterations at the mRNA level. In the rat, administration of amphetamine, methamphetamine, or cocaine rapidly upregulates RGS2 mRNA levels in the striatum ${ }^{47}$ on a rapid time scale similar to the induction of $c$-Fos expression. ${ }^{49}$ Moreover, the upregulation is transient and persists with repeated drug administration, ${ }^{50}$ indicating that RGS2 may be involved in the acute actions of amphetamine. Also, because rapid alterations in RGS2 mRNA levels occur repeatedly with each amphetamine administration, RGS2 may function as a modulator for neuroadaptive changes that lead to dependence and sensitization. ${ }^{50}$

Increases in synaptic dopamine after administration of stimulants probably underlie the rapid changes in RGS2. Both the rat and human gene for RGS2 contain cAMP-sensitive elements ${ }^{51,52}$ (the human gene has a CRE ${ }^{51}$ ) and so dopamine stimulation of adenylyl cyclase via G $\alpha$ s-coupled D1 receptors will lead to an increase in RGS2 message. Indeed, dopamine D1 antagonists prevent the amphetamine-induced upregulation of RGS2 mRNA. ${ }^{53}$ Conversely, activation of the Gai/ocoupled D2 receptor inhibits adenylyl cyclase, leading to decreased RGS2 mRNA expression, whereas D2 antagonists, such as haloperidol, stimulate RGS2 mRNA expression and show an additive effect with amphetamine. ${ }^{47,53}$

The rapid and selective upregulation of RGS2 mRNA in response to stimulants indicates a role for RGS2 in the control of dopamine-mediated signaling events. Although RGS2 preferentially acts at G $\alpha \mathrm{q}$ it can also act as a GAP to regulate G $\alpha 0 .{ }^{54}$ Consequently, increased levels of RGS2 would probably reduce $\mathrm{D} 2$ receptor signaling by this GAP activity. Yet, there is no similar GAP activity at the Goscoupled dopamine D1 receptor. Therefore, the ability of RGS2 to modulate D2 but not D1 signaling could explain the altered balance between D1 and D2 signaling that is thought to underlie adaptive processes to chronic cocaine. On the other hand, RGS2 as well as several other RGS proteins (RGS3, 4,10 , and 13) directly inhibit adenylyl cyclases 2, 5 , and $6 .{ }^{55,56}$ The mechanism behind this effect is unknown but could be due to a direct interaction of RGS2 with Gas, leading to GAP-independent 
inhibition, and/or a direct interaction with the adenylyl cyclase enzyme. ${ }^{57}$ Thus, although RGS2 does not directly modulate D1 receptor signaling by acting as a GAP, it might negatively regulate D1 receptor signaling to adenylyl cyclase.

The rapid control of RGS2 expression by stimulants provides a potential feedback mechanism to finely tune dopaminergic signaling. By acting at an early stage of the signaling cascade, RGS2 can inhibit multiple branches of the D2 receptor signaling cascade as well as D1 receptor signaling to adenylyl cyclase and its downstream effects. In cases where both receptors are coexpressed on the same neuron there is the potential for cross-talk. Consequently, RGS2 can regulate many dopamine-induced behaviors associated with drugs of abuse.

Chronic morphine, acting at Gai/o-coupled $\mu$ opioid receptors to inhibit adenylyl cyclase, has been reported to reduce RGS2 mRNA in dopaminergic neurons in the ventral tegmental area (VTA) of mice. ${ }^{58}$ In contrast, precipitation of opioid withdrawal leads to a modest increase in RGS2 message in the LC. ${ }^{59}$ Chronic administration of the "date rape" drug GHB ( $\gamma$-hydroxybutyrate), acting at $G_{A B A}$ receptors similarly reduces RGS2 mRNA expression in the $\mathrm{VTA},{ }^{58}$ a region wherein $\mathrm{GABA}_{\mathrm{B}}$ receptor coupling to inwardly rectifying potassium channels is sensitive to modulation by RGS2. Therefore, the GHB-induced reduction in RGS2 mRNA might provide positive feedback to enhance GABA coupling efficiency and increase the potency of GHB to inhibit dopamine firing rates. Indeed, GHB-induced decreases in RGS2 mRNA are accompanied by a loss of drinking preference for GHB. ${ }^{58}$

\section{RGS4}

RGS4 is also an unstable protein that is subjected to $\mathrm{N}$-end rule degradation ${ }^{48,60}$ and is regulated by transcription and RNA stabilization. ${ }^{61}$ Addictive drugs generally lead to increased RGS4 mRNA expression, although the effects are not as marked as with RGS2 or as consistent, ${ }^{53,59,62-64}$ which may be due to region and temporally specific changes. In the severely morphine-dependent mouse there is a downregulation of RGS4 mRNA in the lateral hypothalamus. ${ }^{64}$ In the rat, RGS4 protein is upregulated in the NAcc after acute morphine but downregulated in the LC, ${ }^{63}$ where RGS4 protein is expressed mainly in nora- drenergic (tyrosine hydroxylase positive) neurons. ${ }^{59}$ In contrast, treatment of rats with high levels of morphine for several days led to a twofold increase in the level of RGS4 protein in the LC, but with no change in the mRNA for RGS4. ${ }^{59}$ The increased protein expression returned to baseline after precipitation of withdrawal with the opioid antagonist naloxone, accompanied by a threefold increase in RGS4 message. A plausible rationale for this seeming mismatch between RNA and protein is that the protein is stabilized in the presence of chronic morphine such that withdrawal of morphine leads to increase proteolytic breakdown, as a consequence of which the level of mRNA is increased. ${ }^{59}$ Although large doses of morphine and naloxone were used in these studies, no changes were observed in the expression of several other RGS proteins and no changes in RGS4 protein or message were observed in the PAG, an area important for morphine antinociception. On the other hand, RGS4 mRNA was decreased in the occipital lobe after precipitated withdrawal, an effect that was attributed to withdrawal-induced increases in noradrenergic signaling at Gos-coupled stimulatory $\beta$-adrenergic receptors. ${ }^{59}$

In vitro, RGS4 negatively modulates signaling at opioid receptors by acting as a GAP. ${ }^{59,65}$ Therefore, the increased RGS4 seen in the LC after chronic morphine exposure will reduce Gi/o-mediated signaling further and so contribute to tolerance development. However, there are conflicting reports from studies using RGS4-knockout mice of the importance of RGS4. A study of RGS4-knockout mice has reported no morphine phenotype, ${ }^{66}$ which might indicate that other RGS proteins are functionally redundant with RGS4. In contrast, a study with an independent strain of mice has reported increased reward and more severe withdrawal symptoms. ${ }^{67}$

The response of RGS4 mRNA levels to stimulants has been various and assay dependent. ${ }^{50,54,63,68-71}$ Yuferov et al. ${ }^{69}$ reported a decrease in RGS4 mRNA in the caudate putamen of Fisher rats after 3 days of binge cocaine. Similarly, Schwendt et al. ${ }^{71}$ report a decrease in RGS4 mRNA expression in the prefrontal cortex and dorsolateral striatum 21 days after abstinence from cocaine either self-administered or by noncontingent binge pattern administration. Strikingly, exposure of the rats to the cocaineassociated environment caused a return of RGS4 to normal levels, indicating a role for RGS4 in the long-term actions of cocaine. 
Dopamine receptor agonists control RGS4 mRNA levels in a manner that is opposite to that of RGS2. ${ }^{53,68}$ Dopamine D1 agonists decrease RGS4 gene expression and D2 agonists increase RGS4 gene expression. Consequently, observed changes in RGS4 may result from the altered balance of dopamine receptor functioning that occurs after prolonged cocaine where there is an increase in D1 function and a decrease in D2 function. Alternatively, blockade of $N$-methyl-D-aspartate receptors decreases RGS4 mRNA, ${ }^{72}$ suggesting a role for the cocaine-induced downregulation of $N$-methylD-aspartate receptor subunits. With the potential negative modulation by RGS4 of Gai/o-coupled D2 receptors, the reduction in RGS4 may contribute to resetting the balance of dopamine D2 and D1 signaling.

\section{RGS9-2}

Changes in RGS9-2 protein levels after morphine have been reported, but the direction is temporally dependent. Two hours after acute morphine, RGS92 protein levels increase by $50 \%$ in the caudate putamen and NAcc of mice, along with regions involved in the antinociceptive actions of morphine, namely, the PAG and dorsal horn of spinal cord. ${ }^{46,73}$ In contrast, chronic exposure to morphine reverses this rise and ultimately leads to a $50 \%$ reduction in basal RGS9-2 protein levels. No changes in the levels of the binding partner $\mathrm{G} \beta 5$ were observed. ${ }^{73}$

These dynamic changes in RGS9-2 protein seen after morphine administration are not accompanied by alterations in mRNA levels. Therefore, morphine treatment might alter the protein itself, as appears to occur with the smaller R4 family members. This may result from the complex structure of RGS9-2 and its requirement for binding partners, such as Gß5 and R7BP, which confer protein stability and localization as well as promote proper folding of RGS9-2. ${ }^{13-16}$ Interruption or stabilization of these protein binding and folding processes could lead to mRNA-independent changes in RGS9-2 levels. For example, members of the R7 family have a conserved Pro-Glu-Ser-Thr (PEST) sequence in the GGL domain, a signal for protein degradation that may be hidden by the binding of G $\beta 5 .^{74}$

The physiological roles of RGS9-2 protein in the actions of abused drugs have been inferred from studies using targeted gene deletion or antisense oligonucleotides to knock out/knock down RGS9-2 in rodents. RGS9-knockout mice showed more potent responses to morphine related to their abuse potential and other pharmacologies. ${ }^{46}$ For example, in the conditioned place preference assay mice are trained to associate administration of drug with a particular environment as an indication of reward. In this assay morphine is "rewarding" at low doses, although this effect is reversed at higher doses. RGS9-null mice show an approximately 10fold higher potency of morphine in this assay than littermate wild-type controls. The increased potency of morphine is reversed by restoration of RGS9-2 protein levels by injection of virus expressing RGS directly into the in the NAcc. ${ }^{46}$ In contrast, overexpression of RGS4 has no effect, suggesting a selectivity of RGS9-2. Because both RGS9-2 and RGS4 readily act as GAPs for G $\alpha$ o, this selectivity is likely to be achieved by the correct targeting of the more complex RGS9-2 to the $\mu$-opioid receptor.

In mice null for RGS9 ${ }^{46}$ or where RGS9 has been knocked down by antisense treatment, ${ }^{75,76}$ there is an increase in morphine-mediated antinociception and a delay in the development of antinociceptive tolerance. Similarly, knockdown of the R7 family binding partner G $\beta 5$ enhances the antinociceptive response to the $\mu$-opioid receptor agonist DAMGO and inhibits acute tolerance. ${ }^{76}$ These findings suggest that endogenous RGS9-2 inhibits $\mu$-opioid-mediated antinociception. Thus, the increase in RGS9-2 expression after acute morphine might enhance this negative modulation and so contribute to the loss of effect, that is, tolerance. On the other hand, antisense knockdown of RGS9 and G 55 facilitates tolerance to more sustained morphine exposure. ${ }^{75,76}$ The reduction in RGS9-2 after chronic morphine will therefore act to maintain antinociception as tolerance develops, although this could incur the cost of increased dependence. Indeed, compared to wild-type mice, RGS9-knockout mice show a significant increase in behaviors associated with withdrawal including jumping, wetdog shakes, paw tremor, diarrhea, and ptosis, as well as an increased in $c$-Fos expression in the LC. ${ }^{46}$ It appears from studies to date that RGS92 modulates all morphine pharmacology without selectivity.

In contrast to chronic morphine, chronic cocaine has been reported to cause relatively small increases in RGS9-2 protein in the NAcc and caudate 
putamen, particularly in animals that selfadministered the drug. ${ }^{77}$ As observed with morphine there was no change at the mRNA level. In contrast, acute amphetamine has been reported to reduce RGS9 mRNA expression in the dorsal striatum of male rats, ${ }^{47}$ although a second study using direct D1 and D2 agonists showed no effect. ${ }^{53}$ Despite these small changes RGS9-2 does appear to be important for modulating dopamine signaling in striatal regions. RGS9-2 is expressed in dopamine D2 expressing neurons in the NAcc ${ }^{44}$ and acts as a GAP only for $\mathrm{G} \alpha \mathrm{o}$, which is almost exclusively responsible for D2 receptor signaling. ${ }^{78}$ Moreover, mice lacking RGS9 show a dopaminergic-mediated dyskinesia, ${ }^{79}$ and MTPT (1-methyl-4-phenyl-1,2,3,6tetrahydropyridine)-treated monkeys show a dyskinesia that is significantly reduced by viral expression of RGS9 in the dorsal striatum. ${ }^{80}$ Also, monkeys overexpressing RGS9 exhibit a reduced susceptibility to L-dopa-induced dyskinesias. ${ }^{80}$

As a consequence of loss of negative modulation of D2 signaling, RGS9-knockout mice demonstrate increases in cocaine-induced locomotor activity, which can be prevented by overexpression of RGS9-2 in the NAcc. ${ }^{74}$ In addition, these mice show a potentiated locomotor sensitization on repeated cocaine administration; sensitization of stimulantmediated behaviors is considered a major component of drug-seeking behavior. The rewarding effect of cocaine (as measured by place preference) is also increased, although the effect is not as profound as with morphine.

Exposure of ovariectomized female rats to estrogen for 2 weeks has been reported to cause a 30\% decrease in the level of mRNA for RGS9 in the shell of the NAcc. ${ }^{81}$ A resulting reduction in RGS9-2 protein should lead to increased signaling at G $\alpha \mathrm{i} / \mathrm{o}$-coupled receptors. Such a mechanism might explain the increase in dopamine function caused by estrogen and consequently may contribute to the increased vulnerability to the reinforcing effects of drugs of abuse seen in females. ${ }^{82}$

\section{Other RGS proteins}

There are isolated reports linking RGS proteins other than RGS2, 4, and 9 to abused drugs. Several of these results are from microarray analysis profiling after alcohol or nicotine in addition to changes induced by opioids or stimulants.

\section{R4 family}

RGS3 and RGS5 are upregulated after amphetamine in the striatum of the rat, although this requires more sustained stimulation than required to upregulate RGS2. ${ }^{47}$ However, as with RGS2, upregulation of RGS3, but not RGS5, persists with repeated amphetamine administration. ${ }^{50}$ A reduction in RGS13 has been reported in the prefrontal cortex, NAcc, VTA, and amygdala of rats given chronic nicotine. ${ }^{83}$ Nicotine challenge after intermittent exposure and withdrawal resulted in decreased expression of RGS16 in several mouse strains but an increase in a quasi-congenic RQI (recombinant quantitative trait loci introgression) strain. ${ }^{84}$

\section{R7 family}

A small increase in RGS7 protein, after acute, but not chronic, morphine is seen in the LC. ${ }^{59}$ As with knockdown of RGS9, antisense knockdown of other R7 family members RGS6, 7, or 11 gives an increase in morphine-mediated antinociception and a delay in the development of antinociceptive tolerance. ${ }^{76}$ In the $\mathrm{C} 3 \mathrm{H} / \mathrm{HeJ}$ mouse, but not the C57/BL6 mouse, there is a significant regulation of RGS11 in the amygdala and hippocampus in response to chronic nicotine. ${ }^{85}$

\section{RZ family}

RGS20 has been shown to modulate $\mu$-opioid receptor signaling in vitro, ${ }^{86}$ and antisense studies have demonstrated that both RGS19 and RGS20 enhance morphine antinociception in the mouse. ${ }^{87}$ Withdrawal from chronic ethanol in mice showed a downregulation of RGS19-interacting protein, a protein that binds to both RGS19 and G $\alpha$ and targets $\mathrm{G} \alpha$ for degradation. ${ }^{88}$

\section{RGS proteins as potential targets for the treatment of addictive diseases}

Levels of several RGS proteins are manipulated by addictive drugs, and experiments with knockdown/knockout of RGS proteins show that several of these proteins modulate signaling pathways downstream of GPCRs involved in addiction and subsequent behaviors. With the fine-tuning that this interdependency can evoke, it is possible to envisage RGS proteins might be targets for drug abuse treatment. Obviously the study of the role of RGS proteins in addiction would benefit from the 
development of small-molecule inhibitors of RGS proteins. This research is still in its infancy, although there are indications for interaction sites at the RGS-G $\alpha$ interface that could be specific for particular RGS-G $\alpha$ combinations ${ }^{89}$ and that compounds that target $\mathrm{G} \alpha-\mathrm{RGS}$ interactions can be found, either by high-throughput screening ${ }^{90-92}$ or rational design. ${ }^{93}$

An immediate target for intervention, with its distribution and ability to modulate both morphineinduced and cocaine-induced behaviors, is RGS9-2. On the basis of studies in rodents, a compound that stimulates RGS9-2 levels or activity would be expected to decrease stimulant and opioid reward as well as dependence and drug-seeking behavior. Conversely, in the pain clinic, where enhanced analgesic activity of morphine is required, an inhibitor of RGS9-2 activity would be the required target, predicted to enhance the antinociceptive response to morphine and decrease acute tolerance. However, because RGS9-2 appears to modulate all $\mu$-opioidmediated behaviors, such an inhibitor would also increase dependence, reward, and other unwanted actions. An alternative approach might be to use an RGS9-2 inhibitor as a adjunct to a partial agonist (such as buprenorphine) where additional selectivity of action could be obtained based on efficacy. ${ }^{94,95}$

The small RGS proteins, such as RGS2 and RGS4, are less likely targets. One RGS4-knockout mouse strain shows no opioid phenotype, indicating that there may be functional redundancy for GAP activity. Moreover, RGS2 and RGS4 are widely expressed throughout the brain and thus much less likely to provide a selective target, although it is possible that selectivity could be imposed through specific $\mathrm{G} \alpha$ subtype and receptor interactions. Furthermore, RGS4 is important in the parasympathetic control of heart rate, ${ }^{96}$ whereas RGS2 is important for blood pressure regulation. ${ }^{97}$ Interference with these proteins is likely to have serious cardiovascular effects.

Of the more than 30 RGS proteins identified, relatively few have been studied in detail, yet several have been reported to show changes in expression in response to addictive drugs and may be important in specific aspects of the pharmacology of addiction. To study each RGS protein individually is a daunting task. An alternative approach is to use systems that express $\mathrm{G} \alpha$ proteins with a mutation in the conserved RGS binding domain to render them RGS insensitive. ${ }^{98}$ Such systems allow the study of global RGS protein inhibition on receptor signaling. Mice expressing these RGS-insensitive G $\alpha$ proteins are now available. ${ }^{99}$ Such mice will probably show altered pharmacology across a whole spectrum of effects but will at least provide proof of principle that the pharmacology of addictive drugs can be profoundly altered by inhibition of RGS protein GAP activity. We can look forward to learning much more about the roles of these important accessory proteins in the pharmacology of addiction and as possible targets for addictive medications.

\section{Acknowledgments}

I thank the National Institute on Drug Abuse for support (Grant DA04087) and Jeffrey Talbot for critical reading of the manuscript.

\section{Conflicts of interest}

The author declares no conflicts of interest.

\section{References}

1. Berman, D.M. \& Gilman. 1998. Mammalian RGS proteins: barbarians at the gate. J. Biol. Chem. 273: 12691272.

2. Ross, E.M. \& T.M. Wilkie. 2000. GTPase-activating proteins for heterotrimeric $\mathrm{G}$ proteins: regulators of $\mathrm{G}$ protein signaling (RGS) and RGS-like proteins. Annu. Rev. Biochem. 69: 795-827.

3. De Vries, L., B. Zheng, T. Fischer, et al. 2000. The regulator of G protein signaling family. Annu. Rev. Pharmacol. Toxicol. 40: 235-271.

4. Hollinger, S. \& J.R. Hepler. 2002. Cellular regulation of RGS proteins: modulators and integrators of $G$ protein signaling. Pharmacol. Rev. 54: 527-559.

5. Traynor, J.R. \& R.R. Neubig. 2005. Regulators of G protein signaling and drugs of abuse. Mol. Intervent. 5: 3041.

6. Koob, G.F. \& E.J. Nestler. 1997. The neurobiology of drug addiction. J. Neuropsychiatry Clin. Neurosci. 9: 482-497.

7. Koob, G.F., P.P. Sanna \& F.E. Bloom. 1998. Neuroscience of addiction. Neuron 21: 467-476.

8. Kenny, P.J. \& A. Markou. 2004. The ups and downs of addiction: role of metabotropic glutamate receptors. Trends Pharmacol. Sci. 25: 265-272.

9. Tesmer, J.J., D.M. Berman, A.G. Gilman, et al. 1997. Structure of RGS4 bound to AlF4-activated G(i alpha1): stabilization of the transition state for GTP hydrolysis. Cell 89: 251-261. 
10. Clark, M.J., C. Harrison, H. Zhong, et al. 2003. Endogenous RGS protein action modulates mu-opioid signaling through Galphao. Effects on adenylyl cyclase, extracellular signal-regulated kinases, and intracellular calcium pathways. J. Biol. Chem. 278: 9418-9425.

11. Martemyanov, K.A., P.J. Yoo, N.P. Skiba, et al. 2005. R7BP, a novel neuronal protein interacting with RGS proteins of the R7 family. J. Biol. Chem. 280: 51335136.

12. Ballon, D.R., P.L. Flanary, D.P. Gladue, et al. 2006. DEP-domain-mediated regulation of GPCR signaling responses. Cell 126: 1079-1093.

13. Drenan, R.M., C.A. Doupnik, M.P. Boyle, et al. 2005. Palmitoylation regulates plasma membrane-nuclear shuttling of R7BP, a novel membrane anchor for the RGS7 family. J. Cell Biol. 169: 623-633.

14. Song, J.H., J.J. Waataja \& K. A. Martemyanov. 2006. Subcellular targeting of RGS9-2 is controlled by multiple molecular determinants on its membrane anchor, R7BP. J. Biol. Chem. 281: 15361-15369.

15. Witherow, D.S., Q. Wang, K. Levay, et al. 2000. Complexes of the $G$ protein subunit $G \beta 5$ with the regulators of G protein signaling RGS7 and RGS9. Characterization in native tissues and in transfected cells. J. Biol. Chem. 275: 24872-24880.

16. Chen, C.K., P. Eversole-Cire, H. Zhang, et al. 2003. Instability of GGL domain-containing RGS proteins in mice lacking the $G$ protein beta-subunit G $\beta 5$. Proc. Natl. Acad. Sci. USA 100: 6604-6609.

17. Balasubramanian, N., K. Levay, T. Keren-Raifman, et al. 2001. Phosphorylation of the regulator of G protein signaling RGS9-1 by protein kinase A is a potential mechanism of light- and $\mathrm{Ca}^{2+}$-mediated regulation of $\mathrm{G}$ protein function in photoreceptors. Biochemistry 40: 12619-12627.

18. Hollinger, S., S. Ramineni \& J.R. Hepler. 2003. Phosphorylation of RGS14 by protein kinase A potentiates its activity toward Goi. Biochemistry 42: 811-819.

19. Jones, T.L. 2004. Role of palmitoylation in RGS protein function. Meth. Enzymol. 389: 33-55.

20. Popov, S.G., U.M. Krishna, J.R. Falck, et al. 2000. $\mathrm{Ca}^{2+} /$ calmodulin reverses phosphatidylinositol 3,4,5trisphosphate-dependent inhibition of regulators of $\mathrm{G}$ protein signaling GTPase-activating protein activity. $J$. Biol. Chem. 275: 18962-18968.

21. Ishii, M., S. Fujita, M. Yamada, et al. 2005. Phosphatidylinositol 3,4,5-trisphosphate and $\mathrm{Ca}^{2+} /$ calmodulin competitively bind to the regulators of G-protein-signaling (RGS) domain of RGS4 and reciprocally regulate its action. Biochem. J. 385: 65-73.
22. Day, P.W., J.J. Tesmer, R. Sterne-Marr, et al. 2004. Char-

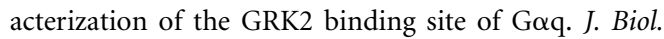
Chem. 279: 53643-53652.

23. Kozasa, T., X. Jiang, M.J. Hart, et al. 1998. p115 RhoGEF, a GTPase activating protein for Galpha12 and Galpha13. Science 280: 2109-2111.

24. Booden, M.A., D.P. Siderovski \& C.J. Der. 2001. Leukemia-associated Rho guanine nucleotide exchange factor promotes G $\alpha \mathrm{q}$-coupled activation of RhoA. Mol. Cell. Biol. 22: 4053-4061.

25. Linseman, D. A., F.A. Loucks \& F. Alexandra. 2008. Diverse roles of Rho family GTPases in neuronal development, survival, and death. Front. Biosci. 13: 657-676.

26. Lan, K.L., H. Zhong, M. Nanamori, et al. 2000. Rapid kinetics of regulator of G-protein signaling (RGS)mediated Galphai and Galphao deactivation. Galpha specificity of RGS4 and RGS7. J. Biol. Chem. 275: 3349733503.

27. Posner, B.A., A.G. Gilman \& B.A. Harris. 1999. Regulators of $G$ protein signaling 6 and 7. Purification of complexes with G $\beta 5$ and assessment of their effects on G protein-mediated signaling pathways. J. Biol. Chem. 274: 31087-31093.

28. Heximer, S.P., N. Watson, M.E. Linder, et al. 1997. RGS2/GOS8 is a selective inhibitor of G $\alpha \mathrm{q}$ function. Biochemistry 94: 14389-14393.

29. Xu, X., W. Zeng, S. Popov, et al. 1999. RGS proteins determine signaling specificity of Gq-coupled receptors. J. Biol. Chem. 274: 3549-3556.

30. Wang, Q., M. Liu, B. Mullah, et al. 2002. Receptorselective effects of endogenous RGS3 and RGS5 to regulate mitogen-activated protein kinase activation in rat vascular smooth muscle cells. J. Biol. Chem. 277: 2494924958.

31. Bernstein, L.S., S. Ramineni, C. Hague, et al. 2004. RGS2 binds directly and selectively to the M1 muscarinic acetylcholine receptor third intracellular loop to modulate Gq/11alpha signaling. J. Biol. Chem. 279: 2124821256.

32. Saitoh, O., Y. Murata, M. Odagiri, et al. 2002. Alternative splicing of RGS8 gene determines inhibitory function of receptor type-specific Gq signaling. Proc. Natl. Acad. Sci. USA 99: 10138-10143.

33. Wang, Q., L.Y. Liu-Chen \& J.R. Traynor. 2009. Differential modulation of $\mu$ - and $\delta$-opioid receptor agonists by endogenous RGS4 protein in SH-SY5Y cells. J. Biol. Chem. 284: 18357-18367.

34. Grafstein-Dunn, E., K.H. Young, M.I. Cockett, et al. 2001. Regional distribution of regulators of G-protein signaling (RGS) 1,2,13,14,16 and GIAP messenger 
ribonucleic acids by in situ hybridization in rat brain. Mol. Brain Res. 88: 113-123.

35. Taymans, J.M., C. Wintermolders, P. Te Reile, et al. 2002. Detailed localization of regulator of $G$ protein signaling 2 messenger ribonucleic acid and protein in the rat brain. Neurosci. 1: 1439-1453.

36. Gold, S.J., Y.G. Ni, H.G. Dohman, et al. 1997. Regulators of G-protein signaling (RGS) proteins; region-specific expression of nine subtypes in rat brain. J. Neurosci. 17: 8024-8037.

37. Nomoto, S., K. Adachi, L-X. Yang, et al. 1997. Distribution of RGS4 mRNA in mouse brain shown by in situ hybridization. Biochem. Biophys. Res. Commun. 241: 281-287.

38. Colantuoni, C., T.M. Hyde, S. Mitkus, et al. 2008. Agerelated changes in the expression of schizophrenia susceptibility genes in the human prefrontal cortex. Brain Struct. Funct. 213: 255-271.

39. Mirnics, K., F.A. Middelton, G.D. Stanwood, et al. 2001. Disease-specific changes in regulator of G-protein signaling 4 (RGS4) expression in schizophrenia. Mol. Psychiat. 6: 293-301.

40. Erdely, H.A., C.A. Tamminga, R.C. Roberts, et al. 2006. Regional alterations in RGS4 protein in schizophrenia. Synapse 59: 472-479.

41. Gu, Z., Q. Jiang \& Z. Yan. 2007. RGS4 modulates serotonin signaling in prefrontal cortex and links to serotonin dysfunction in a rat model of schizophrenia. Mol. Pharmacol. 71: 1030-1039.

42. Granneman, J.G., Y. Zhai, Z. Zhu, et al. 1998. Molecular characterization of human and rat RGS9L, a novel splice variant enriched in dopamine target regions, and chromosomal localization of the RGS9 gene. Mol. Pharmacol. 54: 687-694.

43. Thomas, E.A., P.E. Danielson \& J.G. Sutcliffe. 1998. RGS9: a regulator of $G$ protein signaling with specific expression in rat and mouse striatum. J. Neurosci. Res. 52: $118-124$.

44. Rahman, Z., S.J. Gold, P. Potenza, et al. 1999. Cloning and characterization of RGS9-2, a striatal-enriched alternatively spliced product of the RGS9 gene. J. Neurosci. 19: 2016-2026.

45. Greengard, P., P.B. Allen \& A.C. Nairn. 1999. Beyond the dopamine receptor: the DARPP-32/protein phosphatase-1 cascade. Neuron 23: 435-447.

46. Zachariou, V., D. Georgescu, N. Sanchez, et al. 2003. Essential role for RGS9 in opiate action. Proc. Natl. Acad. Sci. USA 100: 13656-13661.

47. Burchett, S.A., M.L. Volk, M.J. Bannon, et al. 1998. Regulators of $G$ protein signaling: rapid changes in mRNA abundance in response to amphetamine. J. Neurochem. 70: 2216-2219.

48. Bodenstein, J., R.K. Sunahara \& R.R. Neubig. 2007. Nterminal residues control proteasomal degradation of RGS2, RGS4, and RGS5 in human embryonic kidney 293 cells. Mol. Pharmacol. 71: 1040-1050.

49. Graybeil, A.M.N., R. Moratalla \& H.A. Robertson. 1990. Amphetamine and cocaine induce drug-specific activation of the c-fos gene in striosome-matrix compartments and limbic subdivisions of the striatum. Proc. Natl. Acad. Sci USA 87: 6912-6916.

50. Burchett, S.A., M.J. Bannon \& J.G. Granneman. 1999. RGS mRNA expression in rat striatum: modulation by dopamine receptors and effects of repeated amphetamine administration. J. Neurochem. 72: 15291533.

51. Siderovski, D.P., S.P. Heximer \& D.R. Forsdyke. 1994. A human gene encoding a putative basic helix-loophelix phosphoprotein whose mRNA increases rapidly in cycloheximide-treated blood mononuclear cells. DNA Cell Biol. 13: 125-147.

52. Thirunavukkarasu, K., D.L. Halladay, R.R. Miles, et al. 2002. Analysis of regulator of G-protein signaling-2 (RGS-2) expression and function in osteoblastic cells. J. Cell. Biochem. 85: 837-850.

53. Taymans, J.-M., J.E. Leysen \& X. Langlois. 2003. Striatal gene expression of RGS2 and RGS4 is specifically mediated by dopamine DI and D2 receptors: clues for RGS2 and RGS4 functions. J. Neurochem. 84: 1118-1127.

54. Ingi, T., A.M. Krumins, P. Chidiac, et al. 1998. Dynamic regulation of RGS2 suggests a novel mechanism in G protein signaling and neuronal plasticity. J. Neurosci. 18: 7178-7188.

55. Salim, S., S. Sinnarajah, J.H. Kehrl, et al. 2003. Identification of RGS2 and type V adenylyl cyclase interaction sites. J. Biol. Chem. 278: 15842-15849.

56. Roy, A.A., A. Baragli, L.S. Bernstein, et al. 2006. RGS2 interacts with Gs and adenylyl cyclase in living cells. Cell. Signal. 18: 336-348.

57. Sinnarajah, S., C.W. Dessauer, D. Srikumar, et al. 2001. RGS2 regulates signal transduction in olfactory neurons by attenuating activation of adenylyl cyclase III. Nature 409: 1051-1055.

58. Labouebe, G., M. Lomazzi, H.G. Cruz, et al. 2007. RGS2 modulates coupling between $\mathrm{GABA}_{\mathrm{B}}$ receptors and GIRK channels in dopamine neurons of the ventral tegmental area. Nat. Neurosci. 12: 1559-1568.

59. Gold, S.J., M.-H. Han, A.E. Herman, et al. 2003. Regulation of RGS proteins by chronic morphine in rat locus coeruleus. Eur. J. Neurosci. 17: 971-980. 
60. Davydov I.V. \& A. Varshavsky. 2000. RGS4 is arginylated and degraded by the $\mathrm{N}$-end rule pathway in vitro. J. Biol. Chem. 275: 22931-22941.

61. Ni, Y.G., S.J. Gold, P.A. Iredale, et al. 1999. Regionspecific regulation of RGS4 (regulator of G-proteinsignaling protein type 4) in brain by stress and glucocorticoids: in vivo and in vitro studies. J. Neurosci. 19: 3674-3680.

62. Nakagawa, T., M. Mimami \& M. Satoh. 2001. Upregulation of RGS4 mRNA by opioid receptor agonists in PC12 cells expressing mu- or kappa- receptors. Eur. J. Pharmacol. 433: 29-36.

63. Bishop, G.B., W.E. Cillinan, E. Curran, et al. 2002. Abused drugs modulate RGS4 mRNA levels in rat brain: comparison between acute drug treatment and a drug challenge after chronic treatment. Neurobiol. Dis. 10: 334-343.

64. Befort, K., D. Filliol, E. Darcq, et al. 2008. Gene expression is altered in the lateral hypothalamus upon activation of the mu opioid receptor. Ann. N. Y. Acad. Sci. 1129: 175-184.

65. Georgoussi, Z., L. Leontiadis, G. Mazarakou, et al. 2006. Selective interaction between $G$ protein subunits and RGS4 with the C-terminal domains of the $\mu$ and $\delta$-opioid receptors regulate opioid receptor signaling. Cell. Signal. 18: $771-782$.

66. Grillet, N., A. Pattyn, C. Contet, et al. 2002. Generation and characterization of RGS4 mutant mice. Mol. Cell. Biol. 25: 4221-4228.

67. Zachariou, V., W. Renthal, S.J. Gold, et al. 2006. RGS4 modulates morphine reward and dependence. Soc. Neurosci. Abst. 32: 724.

68. Schwendt, M., S.J. Gold \& J.F. McGinty. 2006. Acute amphetamine down-regulates RGS4 mRNA and protein expression in rat forebrain: distinct roles for D1 and D2 receptors. J. Neurochem. 96: 16061615.

69. Yuferov, V., T. Kroslak, K.S. Laforge, et al. 2003. Differential gene expression in the rat caudate putamen after 'binge' cocaine administration: advantage of triplicate microarray assays. Synapse 48: 157-169.

70. Zhang, D., L. Zhang, Y. Tang, et al. 2005. Repeated cocaine administration induced gene expression changes through the dopamine D1 receptors. Neuropsychopharmacology 30: 1443-1454.

71. Schwendt, M., M.C. Hearing, R.E. See, et al. 2007. Chronic cocaine reduced RGS4 mRNA in rat prefrontal cortex and dorsal striatum. Neuroreport 18: 1261-1265.

72. Crespo, J.A., J.M. Oliva, M.B. Ghasemzadeh, et al. 2002. Neuroadaptive changes in NMDAR1 gene expression af- ter extinction of cocaine self-administration. Ann. N. Y. Acad. Sci. 965: 78-91.

73. Psifogeorgou, K., P. Papakosta, S.J. Russo, et al. 2007. RGS9-2 is a negative modulator of $\mu$-opioid receptor function. J. Neurochem. 103: 617-625.

74. Kim, E., T. Arnoult, L. Sellin, et al. 1999. Interaction between RGS7 and polycystin. Proc. Natl. Acad. Sci. USA 96: 6371-6376.

75. Garzón, J., M. Rodríguez-Díaz, A.P López-Fando, et al. 2001. RGS9 proteins facilitate acute tolerance to muopioid effects. Eur. J. Neurosci. 13: 801-811.

76. Garzón, J., A. López-Fando \& P. Sanchez-Blanquez. 2003. The R7 family of RGS proteins assists tachyphylaxis and acute tolerance at $\mu$-opioid receptors. Neuropsychopharmacol. 28: 1983-1990.

77. Rahman, Z., J. Schwarz, S.J. Gold, et al. 2003. RGS9 modulates dopamine signaling in the basal ganglia. Neuron 38: 941-952.

78. Jiang, M., K. Spicher, G. Boulay, et al. 2001. Most central nervous system D2 dopamine receptors are coupled to their effectors by Go. Proc. Natl. Acad. Sci. USA 98: 3577 3582.

79. Kovoor, A., P. Seyffarth, J. Ebert, et al. 2005. D2 dopamine receptors colocalize regulator of G-protein signaling 9-2 (RGS9-2) via the RGS9 DEP domain, and RGS9 knock-out mice develop dyskinesias associated with dopamine pathways. J. Neurosci. 25: $2157-$ 2165.

80. Gold, S.J., C.V. Hoang, B.W. Potts, et al. 2007. RGS92 negatively modulates L-3,4-dihydroxyphenylalanineinduced dyskinesia in experimental Parkinson's disease. J. Neurosci. 27: 14338-14348.

81. Sharifi, J.L., D.L. Brady \& J.I. Koenig. 2004. Estrogen modulates RGS9 expression in the nucleus accumbens. Neuroreport 15: 2433-2436.

82. Becker, J.B. 1999. Gender differences in dopaminergic function in striatum and nucleus accumbens. Pharmacol. Biochem. Behav. 64: 803-812.

83. Kuno, O., J.K. Kane, T. Barrett, et al. 2001. Regionspecific transcriptional response to chronic nicotine in rat brain. Brain Res. 909: 194-203.

84. Vadasz, C., M. Saito, D. O’Brien, et al. 2007. Ventral tegmental transcriptome response to intermittent nicotine treatment and withdrawal in BALB/cJ, C57BL/6ByJ and quasi-congenic RQI mice. Neurochem. Res. 32: 457480 .

85. Wang, J., R. Gutala, Y.Y. Hwang, et al. 2008. Strain-and region-specific gene expression profiles in mouse brain in response to chronic nicotine treatment. Genes Brain Behav. 7: 78-87. 
86. Ajit, S.K., S. Ramineni, W. Edris, et al. 2007. RGSZ1 interacts with protein kinase $\mathrm{C}$ interacting protein $\mathrm{PKC}$ 1 and modulates $\mu$ opioid receptor signaling. Cell. Signal. 19: $723-730$.

87. Garzon, J., M. Rodriguez-Munoz, A. Lopez-Fabnos, et al. 2004. RGSZ1 and GAIP regulate $\mu$-but not $\delta$-opioid receptors in mouse cns: role in tachyphylaxis and acute tolerance. Neuropsychopharmacology 29: 1091-1104.

88. Hashimoto, J.G. \& K.M. Wirren. 2008. Neurotoxic consequenes of chronic alcohol withdrawal: expression profiling reveals importance of gender over withdrawal severity. Neuropsychopharmacology 33: 1084-1096.

89. Soundararajan, M., F.S. Willard, A.J. Kimple, et al. 2008. Structural diversity in the RGS domain and its interaction with heterotrimeric G protein $\alpha$-subunits. Proc. Natl. Acad. Sci. USA 105: 6457-6462.

90. Roman, D.L., J.N. Talbot, R.A. Roof, et al. 2007. Identification of small-molecule inhibitors of RGS4 using a high-throughput flow cytometry protein interaction assay. Mol. Pharmacol. 71: 169-175.

91. Young, K.H., Y. Wang, C. Bender, et al. 2004. Yeast-based screening for inhibitors of RGS proteins. Meth. Enzymol. 389: 277-301.

92. Roof, R.A., K. Sobczyk-Kojiro, A. J. Turbiak, et al. 2008. Novel peptide ligands of RGS4 from a focused one-bead, one-compound library. Chem. Biol. Drug Des. 72: 111119.
93. Jin, Y., H. Zhong, J.R. Omnaas, et al. 2004. Structurebased design, synthesis, and pharmacologic evaluation of peptide RGS4 inhibitors. J. Peptide Res. 63: 141146.

94. Clark, M.J., J.J. Linderman \& J.R. Traynor. 2008. Endogenous RGS proteins differentially modulate full and partial mu agonists at adenylyl cyclase: the role of $\mathrm{G}$ protein kinetics in agonist efficacy. Mol. Pharmacol. 73: 1538-1548.

95. Neubig, R.R. \& D.P. Siderovski. 2002. Regulators of Gprotein signalling as new central nervous system drug targets. Nat. Rev. Drug Discov. 1: 187-197.

96. Cifelli, C., R.A. Rose, H. Zhang, et al. 2008. RGS4 regulates parasympathetic signaling and heart rate control in the sinoatrial node. Circ. Res. 103: 527-535.

97. Heximer, S.P., R.H. Knutsen, X. Sun, et al. 2003. Hypertension and prolonged vasoconstrictor signaling in RGS2-deficient mice. J. Clin. Invest. 111: 445452.

98. Clark M.J. \& J.R. Traynor. 2004. Assays for G-proteincoupled receptor signaling using regulators of G-proteins signaling-insensitive $\mathrm{G} \alpha$ subunits. Meth. Enzymol. 389: 155-169.

99. Huang, X., Y. Fu, R.A. Charbeneau, et al. 2006. Pleiotropic phenotype of a genomic knock-in of an RGSinsensitive G184S Gnai2 allele. Mol. Cell. Biol. 26: 68706879 . 